\title{
ANÁLISIS ESTADÍSTICO DEL ENVEJECIMIENTO EN ESPAÑA. \\ PRINCIPALES INDICADORES DEMOGRÁFICOS PARA EL PERIODO 1975-2016*
}

\section{STATISTICAL ANALYSIS OF AGING IN SPAIN. MAIN DEMOGRAPHIC INDICATORS FOR THE PERIOD 1975-2016}

\author{
MARÍA JOSÉ PÉREZ-FRUCTUOSO** \\ Fecha de recepción: 9 de julio de 2017 \\ Fecha de aceptación: 30 de octubre de 2017 \\ Disponible en linea: 30 de noviembre de 2017
}

\section{Para Citar este articulo/To cite this article}

Pérez-Fructuoso, María José, Análisis estadístico del envejecimiento en España. Principales indicadores demográficos para el periodo $1975-$ 2016, 47 Rev.Ibero-Latinoam.Seguros, 167-190 (2017). https://doi. org/10.11144/Javeriana.ris47.aeee doi:10.11144/Javeriana.ris47.aeee

* Artículo de investigación.

** Doctora Europera en Economía Doctora en Ciencias Económicas y Empresariales. Licenciada en Ciencias Actuariales y Financieras. Licenciada en Ciencias Económicas y Empresariales. Docente de la Universidad a Distancia de Madrid (Madrid Open University, UDIMA). Contacto: mariajose. perez@udima.es 


\section{RESUMEN}

El envejecimiento de la población es un fenómeno que afecta a todos los países desarrollados aunque en el caso de España se presenta con 10 años de retraso respecto al resto de Europa. Dicho envejecimiento se debe fundamentalmente a que el número de nacimientos que se producen no garantiza el reemplazo generacional, lo que da lugar a una disminución de la población a medio plazo, y, simultáneamente, la esperanza de vida es mayor. Desde un punto de vista económico, esto implica que los gastos en pensiones serán mayores, ya que la población de 65 años y más, aumentará y además vivirá durante más años. En este artículo se analizan los factores demográficos que afectan en mayor medida a la situación de envejecimiento de la población española a lo largo de los últimos 41 años.

Palabras clave: Envejecimiento; natalidad; mortalidad; fecundidad; migración. 


\begin{abstract}
The rapid aging of the population is affecting all developed countries, although in Spain it started 10 years later than in the rest of Europe. This aging is mainly due to the fact that the number of births does not not guarantee the generational replacement, which results in a reduction of the population in the medium term, while at the same time life expectancy is higher. From an economic point of view, this implies that pensions expenditure will have to increase, since the population aged 65 and over will rise and will also live for more years. This article analyzes the demographic factors that affect the aging situation of the Spanish population over the last 41 years.
\end{abstract}

Keywords: Ageing; natality; mortality; fertility; migration.

\title{
SUMARIO
}

INTRODUCCIÓN. 1. DEFINICIONES SOBRE LOS CONDICIONANTES DEMOGRÁFICOS DEL ENVEJECIMIENTO. 2. ANÁLISIS DE LOS INDICADORES DE ENVEJECIMIENTO Y ESTRUCTURA DE LA POBLACIÓN ESPAÑOLA PARA EL PERIODO 1975-2016. 2.1. Tasa Bruta de Natalidad y Tasa Bruta de Mortalidad. 2.1.1. Otros indicadores de mortalidad: Esperanza de vida al nacer y esperanza de vida a los 65 años de edad. 2.2. Tasa de Fecundidad y Tasa de Fertilidad. 2.3. Indicadores de Migración. 2.4. Algunos indicadores de estructura de la población relativos al envejecimiento. 2.4.1. Edad media y edad mediana. 2.4.2. Índice de envejecimiento. 2.4.3. Tasa de Dependencia de la población mayor de 64 años. CONCLUSIONES. BIBLIOGRAFÍA 


\section{INTRODUCCIÓN}

La estructura demográfica de un país está condicionada por tres factores fundamentales; los nacimientos, las defunciones y las migraciones. Es evidente que los nacimientos contribuyen a aumentar la población de la misma forma que las defunciones la reducen. En cuanto a las migraciones se pueden producir los dos efectos de aumento o disminución, según se trate de inmigraciones o emigraciones. La situación de España, sin embargo, es de excepción en referencia a los condicionantes mencionados ya que cuenta con una de las tasas de fertilidad más bajas de Europa, una de las esperanzas de vida más altas y unos modelos de migración muy volátiles.

El análisis del envejecimiento de la población de un país es fundamental para entender, por ejemplo, la evolución de su sistema de pensiones, su capacidad de financiarlo y las necesidades y posibilidades de reformarlo (Ayuso, et al. 2014b). En este caso, tiene especial interés analizar exhaustivamente, los factores demográficos que condicionan dicho envejecimiento y que, directa o indirectamente, inciden en el número de personas que cotizan al sistema, y por tanto realizan aportaciones al mismo, y en el número de jubilados, o personas que perciben una contraprestación de jubilación. El envejecimiento tiene también efectos importantes sobre el gasto sanitario per cápita, no solo por el hecho de que aumenta con la edad, sino también porque supone un cambio en el tipo de cuidados requeridos, en el gasto farmacéutico, ya que, en el caso de España, la cobertura de este tipo de gastos en los pensionistas es del 100 por cien, además de que las enfermedades padecidas por este colectivo, así como sus tratamientos, conllevan un importe medio de las recetas más elevado (Lorenzo Carrascosa, L., 2003). En resumen, el envejecimiento de una población implica el mantenimiento de niveles de protección social para un colectivo que crece numéricamente con el tiempo.

En este artículo se realiza un estudio demográfico de la población española durante el periodo de 1975 a 2016 y se analizan las causas del envejecimiento de dicha población así como sus principales características, concluyendo, como se verá a continuación, que el envejecimiento de la población española durante el periodo considerado se produce "desde abajo", consecuencia de una disminución de la natalidad, pero también "desde arriba", derivado de las menores tasas de mortalidad acompañadas de un aumento de la esperanza de vida en las personas de edad avanzada. 
La estructura del artículo es la siguiente. Tras esta primera sección introductoria del tema que va a desarrollarse a continuación, en la sección 2 se definen los factores que condicionan el envejecimiento de un país así como de los medidores estadísticos principales. En la sección 3 se realiza el estudio estadístico a través de los indicadores definidos en la sección anterior, para la serie de datos de la población española entre los periodos 1975 y 2016. Finalmente, la sección 4 presenta las conclusiones más relevantes alcanzadas con la realización del artículo a partir de las proyecciones realizadas de las principales variables analizadas.

\section{DEFINICIONES SOBRE LOS CONDICIONANTES DEMOGRÁFICOS DEL ENVEJECIMIENTO}

Como hemos comentado anteriormente, la composición de una población viene definida, desde un punto de vista demográfico, por la natalidad, la mortalidad, la fecundidad y las migraciones.

La natalidad se refiere a los nacimientos que se producen en el conjunto de la población y se mide a través de la Tasa Bruta de Natalidad. La Tasa Bruta de Natalidad (TBN) o Tasa de Natalidad se calcula como el número de nacimientos registrados en un determinado ámbito geográfico durante un periodo de tiempo y por cada 1000 habitantes,

$$
T B N_{t}=\frac{N_{t}}{P_{t}} 1000
$$

siendo $N_{t}$ el número de nacimientos en el año t asociados a las madres pertenecientes al ámbito geográfico de estudio y $P_{t}$ es la población media residente en dicho ámbito geográfico (INE, 2017a).

En nuestro caso, el ámbito geográfico será el total nacional y el periodo de estudio el anual.

La mortalidad, por su parte, es el número de defunciones ocurridas en un ámbito geográfico y periodo determinados. Su medición se realiza a través de la Tasa Bruta de Mortalidad (TBM) que se define como el total de fallecimientos en el año $t$, de personas pertenecientes a un determinado ámbito geográfico por cada mil habitantes de ese ámbito, 


$$
T B M_{t}=\frac{D_{t}}{P_{t}} 1000
$$

siendo $D_{t}$ el número de fallecimientos registrados en el año $t$ relativos a personas pertenecientes al ámbito geográfico de estudio, el total nacional, y $P_{t}$ es la población media residente en dicho ámbito geográfico en ese año (INE, 2017a). La TBM es la frecuencia con la que se producen las defunciones en un periodo de tiempo determinado, por cada mil habitantes.

En lo referente a la fecundidad, ésta se entiende como la frecuencia de nacimientos que se producen en una población en edad de procrear (INE, 2017a). La medición de esta variable se realiza a través del indicador coyuntural de fecundidad (o Tasa de Fertilidad) e indica el número medio de hijos que tendría una mujer a lo largo de su vida fértil, tradicionalmente comprendida entre 15 y 49 años de edad.

Finalmente, las migraciones son movimientos poblaciones que cruzan un límite geográfico cambiando de residencia habitual (INE, 2017a). La migración es externa cuando el límite geográfico se sitúa entre países, es decir, cuando la residencia habitual de los migrantes cambia de un país a otro, mientras que la migración es interna si los límites que se cruzan se encuentran situados dentro del mismo país. El principal indicador de migración exterior es la Tasa de Migración Neta con el Extranjero o Saldo Migratorio, que se obtiene como el total de migraciones netas (inmigraciones menos emigraciones) con el extranjero, que se realizan en un determinado ámbito geográfico a lo largo de un año $t$ por cada 1000 habitantes de dicho ámbito.

Como consecuencia de las modificaciones que experimentan estas variables demográficas a lo largo del tiempo, se producen cambios que afectan a la estructura y características de una población.

Manteniendo constante la mortalidad y una migración nula, la dinámica demográfica de un país depende exclusivamente de los valores que alcanza la Tasa de Fertilidad (Ayuso, et al. 2014b). Cuando la Tasa de Fertilidad está por encima del nivel de reproducción que, actualmente se sitúa en 2.1 hijos por mujer, la población del país crece de forma 
clásica, dando lugar a una pirámide poblacional tradicional. Si la Tasa de Fertilidad se mantiene estacionaria, la población de un país crece al mismo ritmo que lo hace la mano de obra de dicho país. Finalmente cuando la Tasa de Fertilidad es descendente nos podemos encontrar con diversas situaciones:

- Si la Tasa de Fertilidad es decreciente pero su valor está por encima del nivel de reproducción, la población del país al que se refiere dicha tasa envejecerá y dicho envejecimiento se reflejará en un aumento de las diferentes medidas estadísticas de envejecimiento disponibles (número de personas de edad avanzada, porcentaje de personas de edad avanzada, proporción de personas de edad avanzada sobre la población activa y media de edad de la población). Esto se debe a que las cohortes más jóvenes verán reducido su tamaño con respecto a las cohortes de mayor edad.

- Si la Tasa de Fertilidad descendente se estabiliza en algún nivel por encima de la tasa de reproducción, se producen mayores nivel de envejecimiento en una población nuevamente estable y con un crecimiento menor.

- Si la Tasa de Fertilidad descendente se sitúa por debajo de la tasa de reproducción, la población se reduce (experimenta un crecimiento negativo) y se acentúa el envejecimiento de la misma según las cohortes más jóvenes vayan disminuyendo en relación a las cohortes de mayor edad, que también disminuyen. En este caso la pirámide poblacional se invierte representándose con una base más pequeña y una cabeza mayor. En este caso, el crecimiento negativo de la población podría compensarse con otros fenómenos demográficos tales como saldos positivos en los flujos migratorios.

Cuando se produce una situación de cambio de una Tasa de Fertilidad alta a un valor bajo de la misma, ceteris paribus, da lugar a un envejecimiento de la población que lo experimenta. Y si además, el nivel de estancamiento de la Tasa de Fertilidad se produce a niveles muy inferiores al de reproducción, el efecto del envejecimiento de la población se acentúa de forma notable como sucede en los casos de Europa y más concretamente de España, donde la Tasa de Fertilidad en los últimos años no alcanza el 50\% del nivel de reproducción establecido. 
Otro elemento que condiciona la estructura y la evolución poblacional es el número de fallecimientos, entendido como un factor de reducción de la población. La medida normalmente utilizada para estudiar este elemento es la Tasa de Mortalidad o probabilidad de fallecimiento, definida como el número de fallecimientos, respecto a la población original, que se espera que se produzcan durante el próximo periodo. Esta probabilidad es elevada durante los primeros años de vida, después del nacimiento, para luego permanecer constante durante un tiempo hasta que empieza a aumentar con la edad (Ayuso, et al., 2014a). La probabilidad de supervivencia es la probabilidad complementaria de la de fallecimiento, y mide la proporción de individuos de una determinada cohorte de edad que sobreviven hasta el final de un determinado año. En el momento del nacimiento, la suma de todas las probabilidades de supervivencia da lugar a la esperanza de vida, otro concepto fundamental en el estudio demográfico de un país. La esperanza de vida al nacimiento se define como el número medio de años que vivirán los individuos de una determinada cohorte en cada edad en función del patrón de mortalidad observada en las personas de una determinada zona geográfica a lo largo del periodo $t$. Dicho de otra forma, la esperanza de vida al nacer es la media de edad que un individuo de una cohorte de edad puede esperar vivir. Adicionalmente, considerando que el límite establecido en los países más desarrollados para la edad avanzada se sitúa en los 65 años de edad, resulta interesante analizar la influencia en la dinámica población que tiene la esperanza de vida a los 65 años o el número medio de años que vivirá la cohorte de personas que hoy tienen 65 años cumplidos.

Finalmente debemos hacer referencia a las migraciones, ya que dependiendo de sus características, pueden afectar de forma significativa a la estructura poblacional de un país (Ayuso et al., 2014c). Los aspectos a analizar de esta componente poblacional se centran en determinar si la migración neta es relevante, si los migrantes llegan a los países cuando son jóvenes y si se marchan cuando llegan a la edad de jubilación, ya que en ambos casos se produce un rejuvenecimiento de la población afectada, la temporalidad de la estancia en el país receptor, que da lugar a diferentes a migraciones estacionales, temporales o permanentes, $\mathrm{y}$, finalmente, si la migración que se realiza es por motivos laborales, familiares o por otro tipo de motivos como la solicitud de asilo, etc. Para estudiar el fenómeno migratorio español, se analizaran los siguientes indicadores (INE, 2017a): 
- Saldo Migratorio (SM), o diferencia entre las entradas por migración y las salidas por el mismo motivo, para un ámbito geográfico determinado. Si el signo es positivo indica que las entradas superan las salidas y si el saldo es negativo las salidas superan a las entradas de individuos.

- Tasa de Migración Bruta con el Extranjero (TMBE), definida como el total de inmigraciones y emigraciones que realizan con el extranjero durante el año $t$ los individuos de una determinada zona geográfica por cada 1.000 habitantes de dicha zona.

- Tasa de Migración Neta con el Extranjero (TMNE), entendida como el conjunto de inmigraciones menos emigraciones que realizan con el extranjero durante el año $t$ los individuos de una determinada zona geográfica por cada 1000 habitantes de dicha zona.

\section{ANÁLISIS DE LOS INDICADORES DE ENVEJECIMIENTO Y ESTRUCTURA DE LA POBLACIÓN ESPAÑOLA PARA EL PERIODO 1975-2016}

\subsection{Tasa Bruta de Natalidad y Tasa Bruta de Mortalidad}

La Tasa Bruta de Natalidad (TBN) en el año 2016 fue de 8.752481 nacimientos por cada mil habitantes, lo que supuso 0.238910 nacimientos menos que el año anterior, un $2.657 \%$ en términos porcentuales (INE, 2017b).

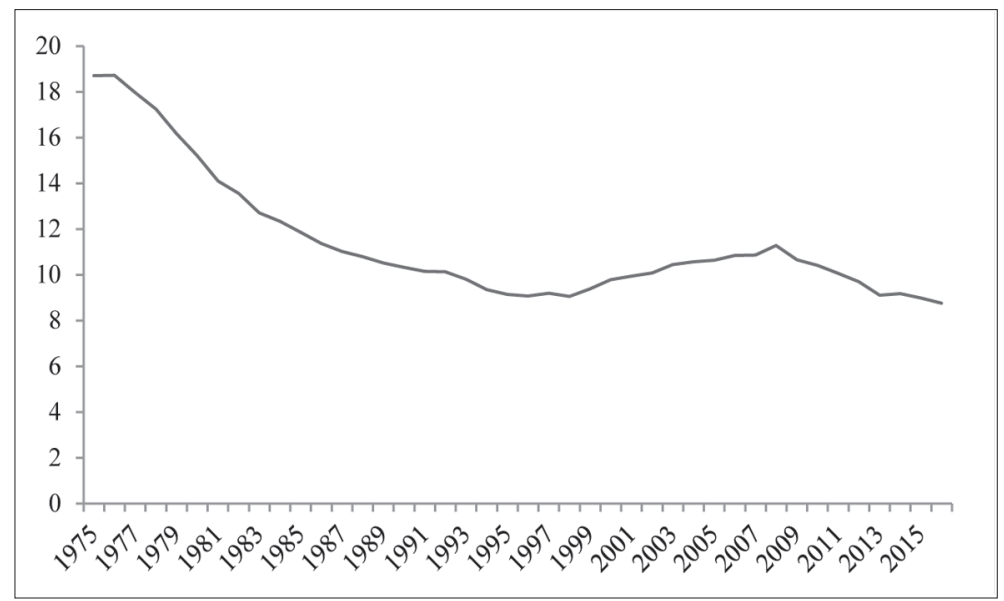

Gráfico 1: Tasa Bruta de Natalidad (TBN) Española. Periodo 1975-2016 Fuente: Elaboración Propia a partir de datos del INE 
Analizando el periodo 1975-2016, la TBN en España muestra una tendencia decreciente con un ligero repunte durante los años de 1999 a 2008, aunque en niveles muy inferiores a los del principio de la serie analizada. Se produce, por tanto, una crisis de natalidad a finales de los años 70, en 1979 concretamente, cuando la TBN empieza a disminuir, que se alarga hasta finales de los años 90. A partir del año 2008 la TBN vuelve a decrecer hasta alcanzar su nivel más bajo en el año 2016. En términos absolutos, desde el año 2008, momento en el que, con una TBN del $11.2759 \%$ y 519779 nacimientos, se alcanza un máximo histórico en tres décadas, el número de nacimientos ha descendido un $21.43 \%$. En 2016, ha habido un total de 408384 nacimientos, que supone el menor valor registrado desde el 2002. Es evidente que si la TBN es baja para un mismo valor de la población media residente, es porque el número de nacimientos a lo largo del tiempo es menor y por tanto, la población analizada estará sufriendo un envejecimiento y el peso de los colectivos de personas mayores será cada vez mayor.

La variación relativa anual de la TBN muestra un comportamiento completamente oscilante, con una línea de tendencia parabólica, siendo la variación media anual de los 40 años analizados (1976-2016) del 1.789\%.

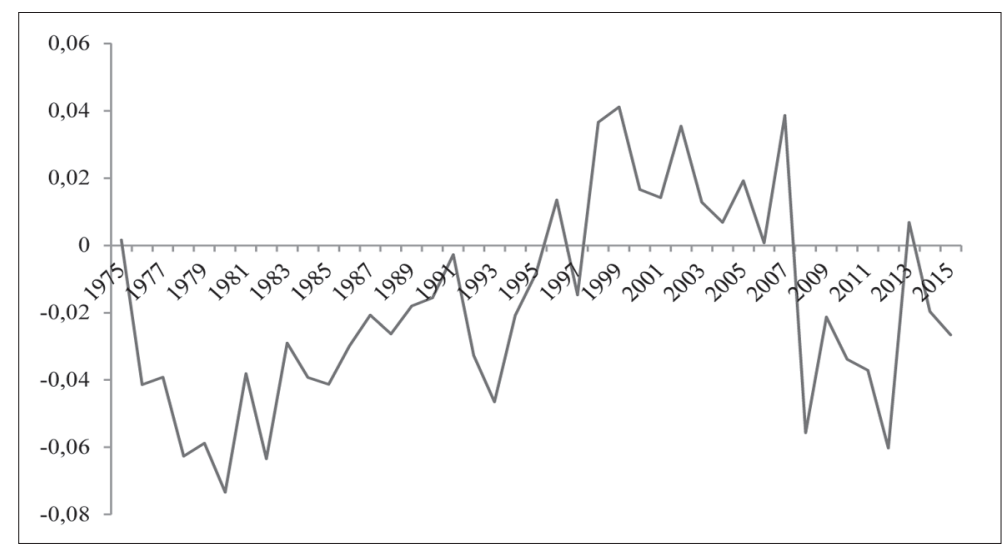

Gráfico 2: Variación Relativa Anual de la TBN Española. Periodo 1975-2016 Fuente: Elaboración propia a partir de datos del INE

Por lo que respecta a la mortalidad, como puede observarse en el Gráfico 3 a continuación, se ha mantenido bastante estable en el periodo de análisis considerado.

En el año 2016, la TBM fue de 8.758056 fallecidos por cada mil habitantes, ligeramente inferior a la del año 2015, que se situó en 9.050714 fallecidos 


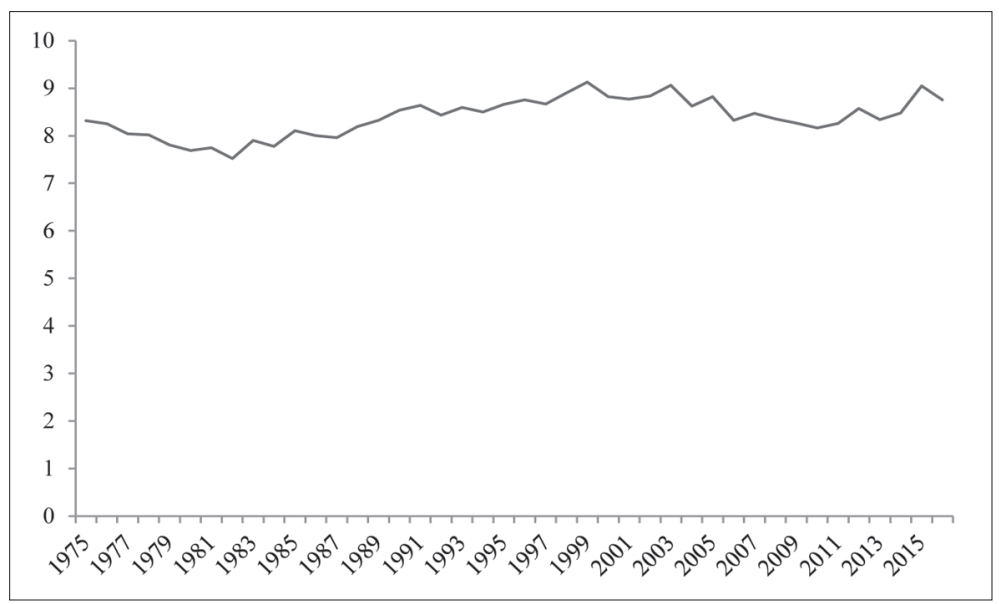

Gráfico 3: Tasa Bruta de Mortalidad (TBM) Española. Periodo 1975-2016 Fuente: Elaboración propia a partir de datos del INE

por cada mil habitantes, y a un nivel muy similar a la TBM de 2014. En total, el número de defunciones en 2016 ascendió a 409099 personas (INE, 2017b).

Analizando el periodo 1975-2016, la TBM en España muestra una tendencia bastante plana entorno al valor de 8 defunciones por cada mil habitantes, si bien es cierto que durante el año 2015 la tasa anual de variación de las defunciones aumentó un $6.7 \%$ respecto al año anterior (en términos absolutos, 26446 defunciones más). Durante el año 2016, la tasa anual de variación de las defunciones volvió a disminuir un 3.2\% respecto a los datos de 2015 con 13177 defunciones menos.

La variación relativa anual de la TBM muestra una línea de tendencia completamente plana, siendo la variación media anual de los 41 años analizados (1975-2016) del 0.161281\%. Esta variación casi nula de la TBM junto con la disminución de la TBN en el periodo considerado, redunda en un envejecimiento de la población en España provocado fundamentalmente por las menores tasas de natalidad.

El estudio conjunto de la evolución de la TBN y de la TBM, muestra una importante caída de la natalidad a partir de 1975, momento en el que comienza a descender sustancialmente hasta colocarse, en 1999, al mismo nivel que el indicador de la tasa de mortalidad (TBM), que se mantiene más o menos estable a lo largo de todo el periodo considerado. Posterior- 


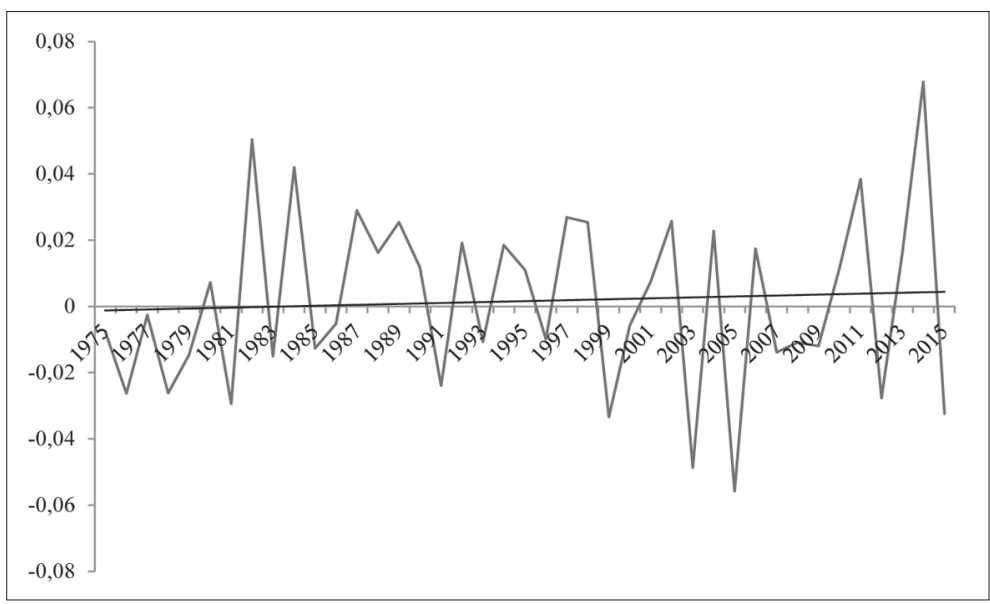

Gráfico 4: Variación Relativa Anual de la TBN Española. Periodo 1975-2016 Fuente: Elaboración propia a partir de datos del INE

mente, la TBN presenta un periodo de crecimiento respecto a la TBM de unos 9 años (2000-2008), para volver a descender desde entonces hasta el año 2015 en que la TBN se sitúa por debajo de la TBM. Esto significa que, por primera vez en los años considerados y desde que se dispone de datos anuales, la diferencia entre los nacimientos y las defunciones es negativa. Durante el año 2016 hubo 408384 nacimientos frente a 409099 defunciones, lo que implica que durante ese año fallecieron 715 personas más de las que nacieron. El Crecimiento Vegetativo, definido como la diferencia entre los nacimientos de madre residente en España y defunciones de residentes en el mismo país, también fue negativo en 2016 y ascendió a 259 personas.

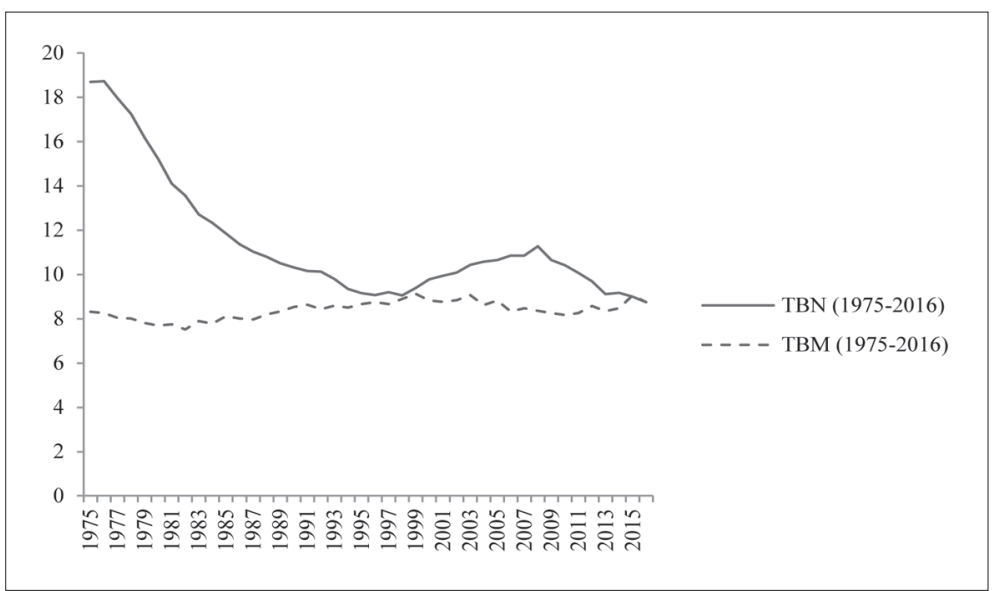

Gráfico 5: Evolución de la TBN y TBM Española. Periodo 1975-2016 Fuente: Elaboración propia a partir de datos del INE 


\subsubsection{Otros indicadores de mortalidad: Esperanza de vida al nacer y esperanza de vida a los 65 años de edad}

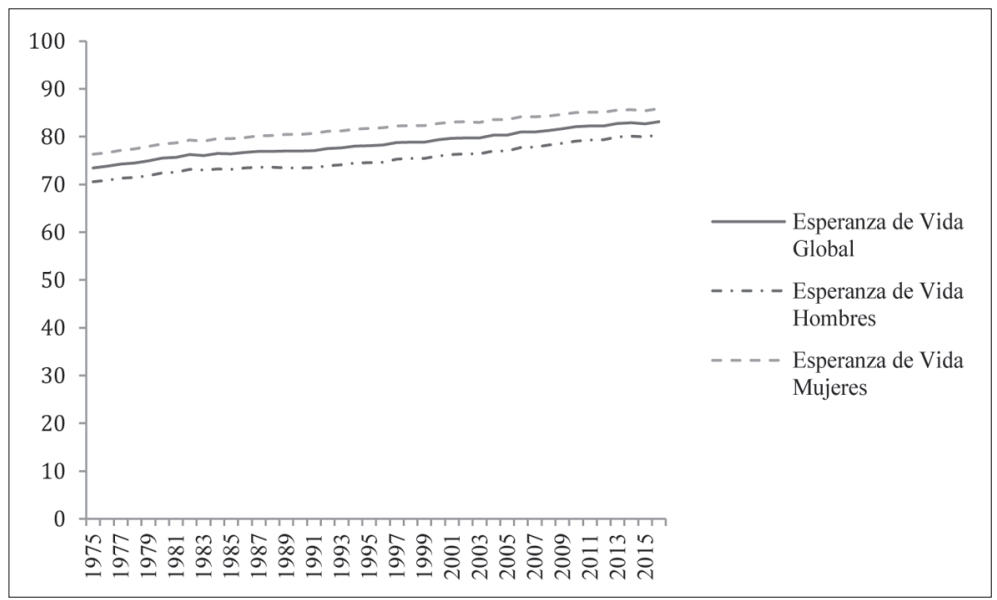

Gráfico 6: Esperanza de Vida al Nacer en España. Periodo 1975-2016 Fuente: Elaboración propia a partir de datos del INE

La esperanza de vida al nacer ha ido creciendo progresivamente desde 1975 hasta la actualidad (INE, 2017b). De los 73.4 años de esperanza de vida conjunta en 1975 hemos pasado a vivir 9.53 años más en 2016, alcanzando, en global, 83.2 años. Analizando este indicador por sexos, en los hombres, la esperanza de vida se sitúa en 80.4 años aumentando en 9.8 años respecto a los 70.5 de 1975 y, en las mujeres, la esperanza de vida hoy es de 85.9 años lo que supone un incremento de 9.61 años respecto de la esperanza registrada en 1975 (76.29 años).

Si consideramos la esperanza de vida a los 65 años, con las condiciones de mortalidad actuales, un hombre que llegue con vida a cumplir esa edad, espera vivir 19.2 años más, mientras que una mujer con 65 años cumplidos tiene una esperanza de vida de 23.1 años más. En promedio, la esperanza de vida a los 65 años para ambos sexos es de 21.34 años, 6.2 años mayor que en 1975.

\subsection{Tasa de Fecundidad y Tasa de Fertilidad}

El número de nacimientos es el flujo de entrada más importante en la estructura demográfica de un país. Y como este número está íntimamente 
relacionado con la estructura demográfica existente (número de mujeres en edad fértil, etc.), su análisis se realizará a través de la Tasa de Fertilidad o indicador coyuntural de fecundidad.

Resulta evidente, además, que la Tasa de Natalidad analizada en el epígrafe anterior presenta importantes vínculos con las tasas de fertilidad $\mathrm{y}$ de fecundidad que vamos a estudiar a continuación.

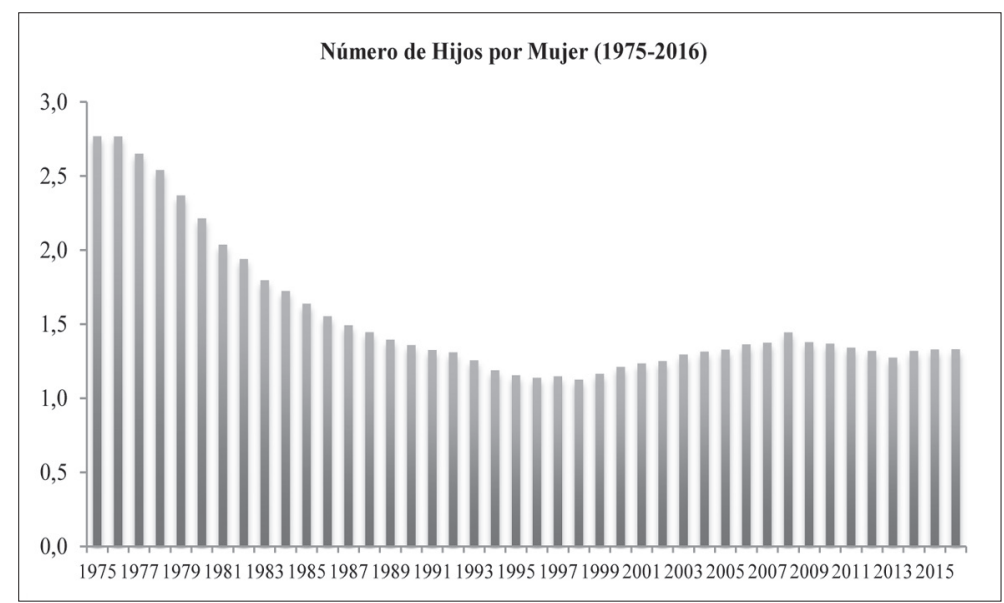

Gráfico 7: Tasa de Fertilidad Española. Periodo 1975-2016

Fuente: Elaboración propia a partir de datos del INE

Como se observa en el Gráfico 7, el número medio de hijos que tendría una mujer de edad comprendida entre 15 y 49 años (edad fértil) es la Tasa de Fertilidad o el indicador coyuntural de fecundidad (INE, 2017b). Este número se ha reducido considerablemente desde los años de origen del estudio, pasando de 2.768481 en 1975 a 1.330801 en 2016, descenso que coincide con el apuntado anteriormente para la Tasa Bruta de Natalidad. A pesar del ligero repunte experimentado en el año 2008, en el que el indicador de fecundidad se elevó, tras más de 20 años de caída, hasta 1.444423, las cifras asociadas a los últimos años de la serie analizada manifiestan de nuevo una tendencia a la baja que se aleja, de forma considerable, del valor 2.1 hijos, establecido como referencia para garantizar la reproducción de una población. Este cambio de comportamiento registrado a partir de 2008, y coincidiendo con el inicio de la crisis económica, se pone también de manifiesto en el número de nacimientos a partir de ese momento, como veremos a continuación. 
Comparando esta Tasa de Fertilidad con la del resto de países europeos (ver Gráfico 8), se pone de manifiesto que España es uno de los países con menores tasas de fertilidad del conjunto de países analizado junto con Italia o Grecia y solo por encima de países como Polonia, Rumania, Lituania, Ucrania, Letonia, Montenegro y Kosovo.

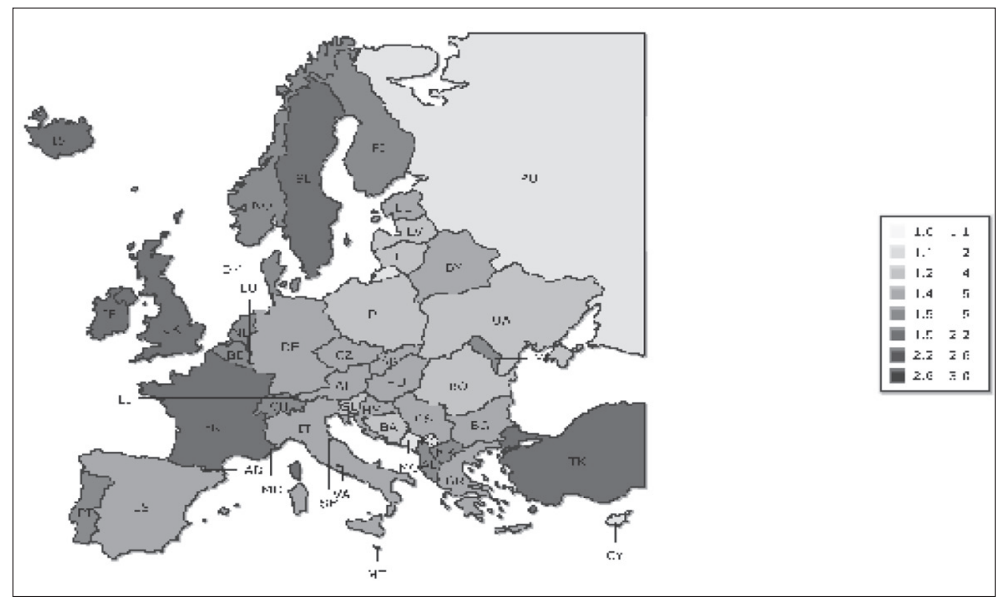

Gráfico 8: Tasas de Fertilidad en Europa

Fuente: CIA World Factbook

http://www.indexmundi.com/map/?t=0\&v=31\&r=eu\&l=es (datos a 1 Enero de 2014)

Respecto al número de nacimientos, se observa también una tendencia decreciente desde el periodo origen del estudio. A partir de 1975, el número de nacimientos se ha reducido un $37.388 \%$, pasando de 669378 en dicho año hasta 408384 en 2016 . Solo el año 2008 presenta un máximo histórico en 30 años, con 519779 niños nacidos. Desde entonces, el descenso registrado en el número de nacimientos es del $21.4312 \%$.

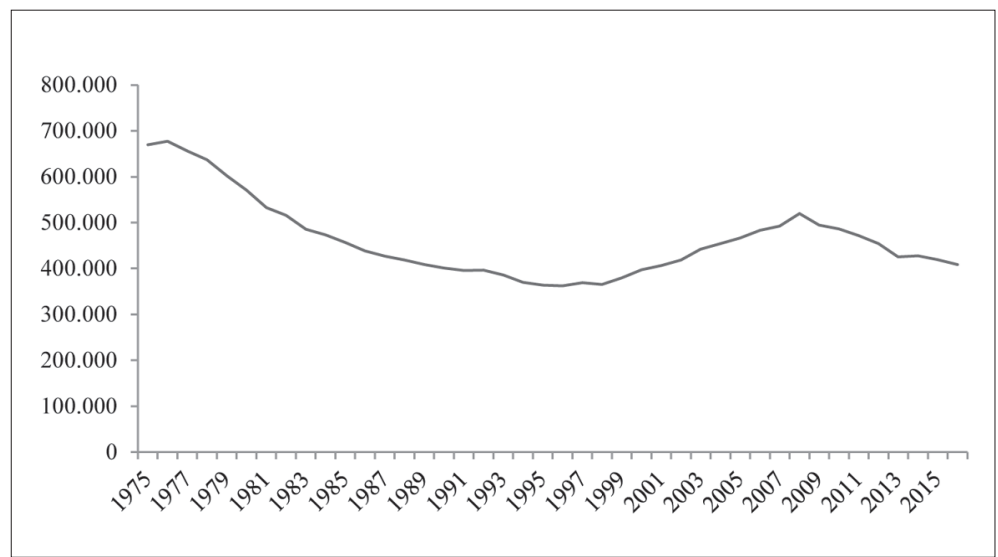

Gráfico 9: Número de nacimientos en España. Periodo 1975-2016 Fuente: Elaboración propia a partir de datos del INE 
La edad media de la maternidad, por su parte, ha ido aumentado progresivamente desde los 29 años (28.85) en 1975, hasta los 32 en 2016 (INE, 2017b).

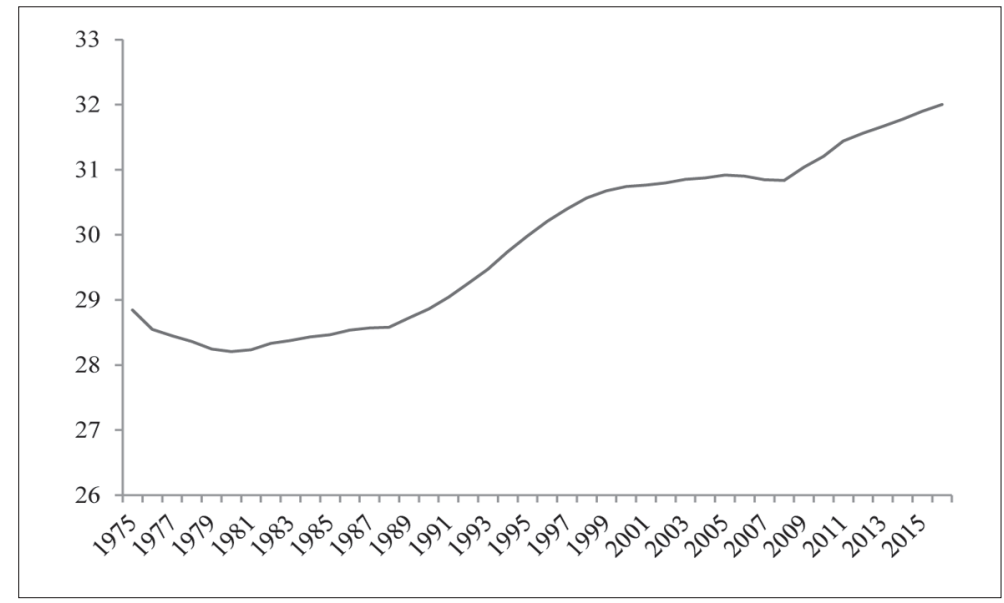

Gráfico 10: Edad Media a la Maternidad en España. Periodo 1975-2016 Fuente: Elaboración propia a partir de datos del INE

El número de mujeres en edad fértil (entre los 15 y los 49 años), ha sido creciente hasta el año 2009 y desde ese momento muestra una tendencia a la baja (10.6 millones en 2016 frente a los 11.6 millones existentes en 2009) principalmente por tres razones: a esa edad llegan menos mujeres nacidas en la crisis de natalidad experimentada durante los años 80 y primera mitad de los 90; hay menos inmigración exterior y hay más emigraciones al exterior.

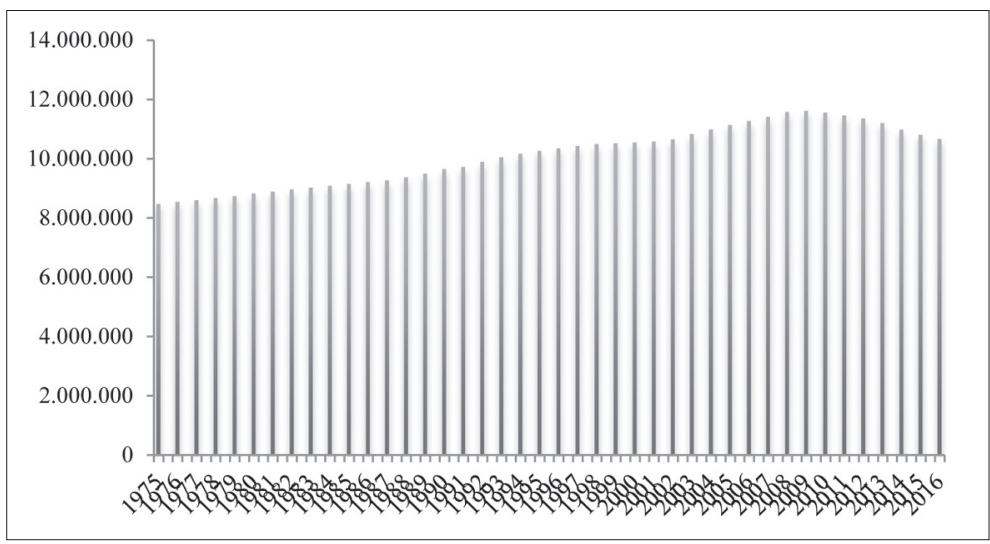

Gráfico 11: Mujeres en Edad Fértil en España. Periodo 1975-2016 Fuente: Elaboración propia a partir de datos del INE 
Como consecuencia de ello, y como se ha puesto de manifiesto en los párrafos anteriores, aunque el número de nacimientos en 2016 se ha reducido respecto de años precedentes, el número medio de hijos por mujer ha aumentado debido al descenso experimentado en el número de mujeres en edad fértil.

\subsection{Indicadores de Migración}

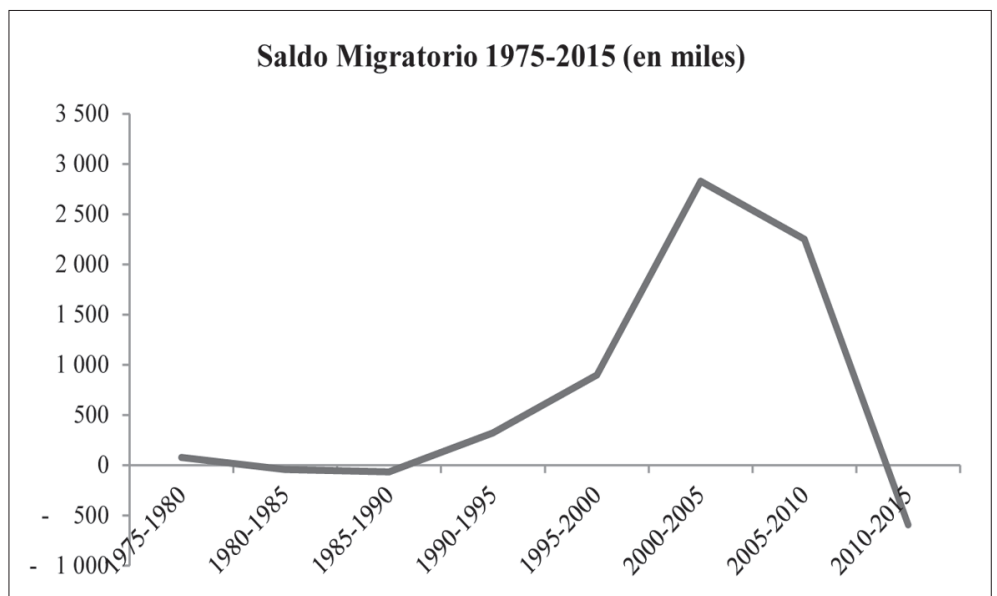

Gráfico 12: Saldo Migratorio en España. Periodo 1975-2016 Fuente: Elaboración propia a partir de datos de la ONU 2015

Como pone de manifiesto el Gráfico 12, España pasó de ser un país de emigrantes hasta la década de los 80 del siglo pasado, a ser un país típicamente de inmigración (INE, 2016a y 2016c). El Saldo Migratorio, definido como la diferencia entre los inmigrantes y emigrantes de un país, pasó de tomar valores negativos, o muy cercanos a cero, a crecer de forma exponencial, especialmente durante el periodo de 2000 a 2008, coincidiendo con la época de crecimiento económico y el auge inmobiliario y de la construcción. Es importante señalar en este punto que la mayor parte de la población que entraba en España hasta el año 2008 estaba incluida en la franja de edad legal para trabajar. Con el inicio de la crisis económica se produce un cambio significativo en el sentido de las migraciones en España, reduciéndose de forma considerable el número de personas procedentes de otros países que vienen a vivir a España y aumentando progresivamente el número de personas españolas que fijan su residencia habitual en otro país. 


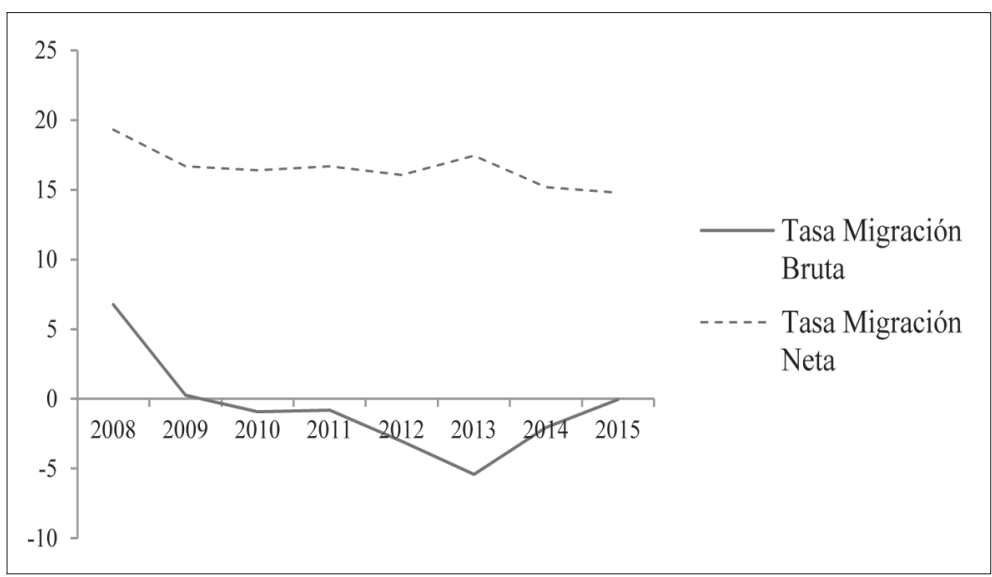

Gráfico 13: Tasas de migración bruta y neta en España. Periodo 2008-2015 Fuente: Elaboración propia a partir de datos del INE

En el Gráfico 13 de las Tasas de Migración, se observa claramente un descenso de las inmigraciones y un aumento de la emigraciones a partir del año 2008, que dan lugar a un Saldo Migratorio negativo a partir del año 2009 y hasta el 2015, momento en el que parece empezar a recuperarse la economía y se frenan las salidas al exterior, tanto de personas nacidas en España como de personas extranjeras residentes en España que vuelven a sus lugares de origen (INE, 2016a).

A 1 de enero de 2016 la población española estaba formada por 46445828 habitantes de los cuales 4418157 eran extranjeros (INE, 2016c). Esta cifra supone una reducción respecto a los datos registrados el 1 de enero del año anterior de 3737 personas. Cabe destacar, sin embargo, que la población con nacionalidad española aumentó durante el año 2015 en 32459 personas como consecuencia de la adquisición de la nacionalidad. En global, el Saldo Migratorio fue negativo, registrándose 36196 inmigrantes menos.

\subsection{Algunos indicadores de estructura de la población relativos al envejecimiento}

\subsubsection{Edad media y edad mediana}

La edad media de la población es un indicador, junto con la edad mediana, de la estructura poblacional, y puede definirse como la media de edades 
de los individuos pertenecientes a un determinado ámbito geográfico a uno de enero del año $t$ (INE, 2017). Una análisis de la evolución de esta edad media, muestra un aumento absoluto de 9.727993 años pasando de vivir (en promedio) 32.995211 años en 1975 a vivir 42.723204 años en 2016. Diferenciando por sexos, los hombres han pasado de 31.723034 años en 1975 a 41.419145 años en 2016. Y para las mujeres, la variación ha ido desde 34.216176 años en 1975 hasta los 43.981636 años de edad en 2016.

La edad mediana es otro indicador del grado de envejecimiento de la estructura por edades de la población. Entendiendo la edad mediana como aquella que divide a la distribución por edades de la población en dos grupos iguales a uno de enero del año $t$ (de forma que la mitad de la población tiene una edad menor o igual que la mediana y la otra mitad tiene una edad mayor o igual a la mediana), su comportamiento ha sido similar al de la edad media. El incremento absoluto experimentado por dicha medida estadística ha sido de 12.765918 años, de forma que en 1975 la mediana de la distribución de edades era de 30.021420 años mientras que en 2016 el valor de la edad mediana era de 42.787338 años.

Comparando ambas medidas estadísticas de centralización, para toda la serie temporal analizada, los valores de la media son superiores a los valores de la mediana, lo que indica una concentración de las edades a la derecha de los valores medios considerados. Es decir, en la distribución predominan las edades por encima de 42 años, otro claro indicio de envejecimiento de la población.

\subsubsection{Indice de envejecimiento}

El porcentaje de individuos de una población mayores de 64 años mide el peso relativo de las personas mayores sobre la población total.

La Tasa de Envejecimiento indica el porcentaje que representa la población de edad mayor de 64 años sobre la población menor de 16 años a principios de un determinado año $t$ (INE, 2017a). Representa, por tanto, el número de personas de edad avanzada por cada 100 personas menores de 16 años. Este índice muestra el envejecimiento actual y el posible envejecimiento futuro de la población. 


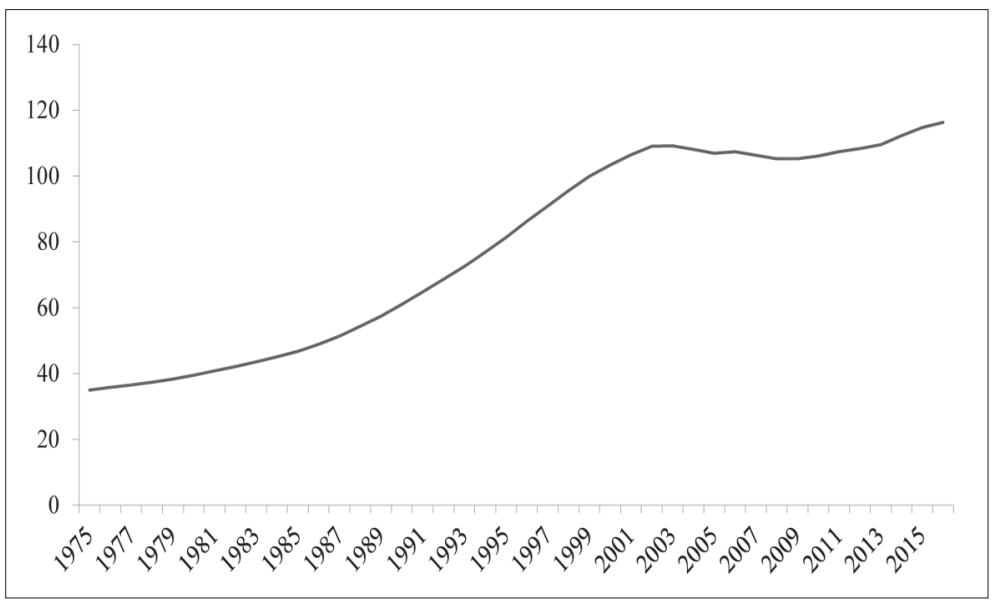

Gráfico 14: Índice de envejecimiento de la población española. Periodo 1975-2016

Fuente: Elaboración propia a partir de datos del INE

Como se observa claramente en el Gráfico 14, este índice muestra una tendencia creciente a lo largo de los años, como consecuencia, fundamentalmente, de una esperanza de vida cada vez mayor, unido a unas mejores condiciones de vida y de salud. En la actualidad, el índice se sitúa en $116.3564 \%$, un $81.369714 \%$ superior que en el año 1975 cuando por cada 100 personas de 16 años, solo había 34.986730 personas mayores de 64 años.

Según los datos proporcionados por el Padrón Continuo del Instituto Nacional de Estadística, a 1 de enero de 2016 hay 8657705 personas mayores (65 y más años), lo que representa el 18.4\% sobre el total de la población (46557008) (Abellán García et al., 2017). Diferenciando por sexos, cabe destacar el hecho de que hay un $32.9 \%$ más de mujeres (4940008) que de hombres (3717697) de edad avanzada.

\subsubsection{Tasa de Dependencia de la población mayor de 64 años}

Este indicador muestra la relación entre la población mayor de 64 años y la población de entre 16 y 64 años, ambas magnitudes referidas a una determinada zona geográfica a principios del año $t$ (INE, 2017a). 


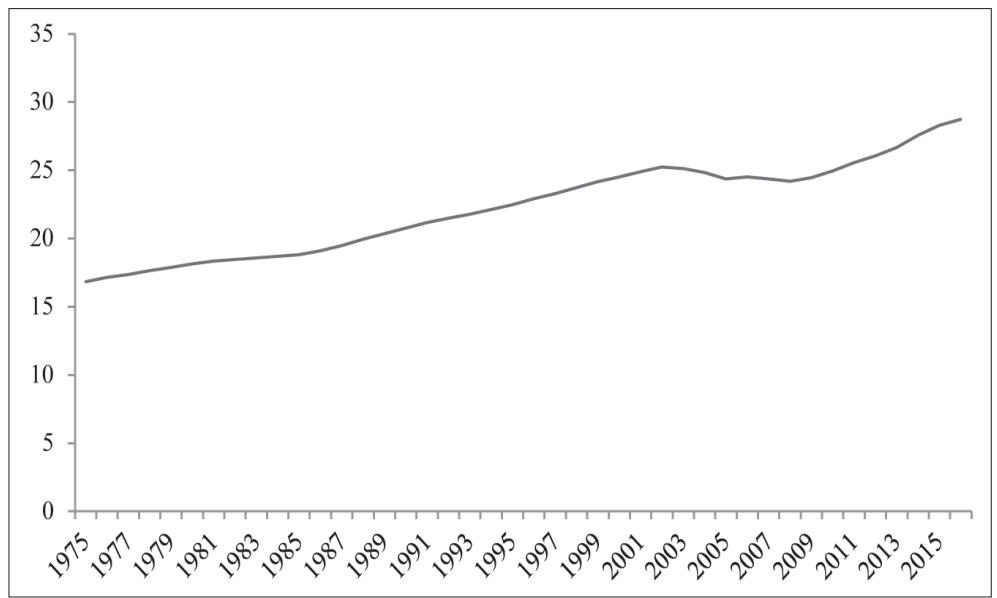

Gráfico 15: Tasa de dependencia de la población mayor de 64 años (1975-2016) Fuente: Elaboración propia a partir de datos del INE

La Tasa de Dependencia de los mayores de 64 años presenta un suave pero contante ascenso, solo interrumpido durante los años 2004-2010, habiéndose elevado en global casi un 60\% entre 1975 y 2016. Este incremento indica una mayor población dependiente, en este segmento de edad, derivada de la reducción de la Tasa de Natalidad y del aumento de la esperanza de vida, de forma que en el año 2052 la población mayor de 64 años constituirá el 37\% de la población total y la Tasa de Dependencia llegará a ser de casi el 100\% según estimaciones realizada por el Instituto Nacional de Estadística (INE, 2016d). Para el año 2016, la tasa de dependencia se sitúa en el 28.741924\%.

\section{CONCLUSIONES}

El envejecimiento que vive la población española se inició en la década de los 50 del siglo pasado, situándose entonces por debajo de la media europea, y los valores proyectados de las diferentes variables que en él influyen auguran que dicho envejecimiento seguirá aumentando en los próximos años. En este sentido, en las mismas condiciones que las actuales, iniciadas en el año 2009, el número de nacimientos es previsible que se reduzca de forma considerable. Así, los datos de las proyecciones demográficas realizadas por el INE siguen mostrando una tendencia decreciente, situándose en el año 2066 por debajo de los 300000 nacimientos (294003). Entre 2016 y 2030 habría un $22 \%$ menos de nacimientos 
que en los 15 años precedentes (lo que supondría 5300000 niños menos). En el año 2031 habría entonces 335937 nacimientos lo que supondría un 17.9\% menos que en 2016.

Este descenso en el número de nacimientos se debería, fundamentalmente, a la importante reducción del conjunto de mujeres en edad fértil cuyo valor se situaría en 9 millones aproximadamente en 2031 y en 7.3 millones en 2066, lo que representa 1.6 millones (o un $15.1 \%$ ) y 3.3 millones (o un 31.13\%) menos que en 2016.

Respecto al número de fallecimientos, se prevé que siga creciendo con el tiempo debido a este fenómeno del envejecimiento, registrándose 595979 defunciones en el año 2065. Entonces, como consecuencia de la bajada de la natalidad y del aumento de la mortalidad en el periodo proyectado, en España se produciría un crecimiento vegetativo negativo durante los próximos 50 años, siendo en todos ellos superior el número de defunciones al de nacimientos.

La estructura poblacional será sufrirá un cambio sustancial de forma que los mayores serán el doble que en la actualidad y triplicaran la cifra de niños. El sexo predominante a la vejez será el femenino, produciéndose una mayor supervivencia de las mujeres frente a una mayor tasa de mortalidad de los hombres.

En lo referente a la fecundidad de las mujeres, las proyecciones para los próximos 50 años prevén una ligera tendencia al alza pasando de 1.33 hijos por mujer en la actualidad a 1.38 al final del periodo de proyección establecido. De igual forma, la edad media a la maternidad también se espera que siga aumentando hasta el año 2066, momento en el que se situaría en los 33 años, 1.1 años por encima del valor actual de 31.9 años.

Mirando al futuro, la esperanza de vida al nacer llegaría a alcanzar, en el año 2031, los 83.2 años en los hombres y los 87.7 años en las mujeres, 3.3 y 2.3 años más, respectivamente, que los valores de esperanza de vida actuales. Para el año 2065, la esperanza de vida de los hombres alcanzaría los 88.5 años y la de las mujeres los 91.6 años de edad. 
Si se mantienen las condiciones actuales, en el año 2065, una mujer de 65 años vivirá, en promedio, otros 28.2 años más y un hombre lo hará 25.1 años más frente a los 22.7 y 18.8 años, respectivamente, que se espera que vivan actualmente.

Finalmente, en lo referente a las proyecciones de migración, suponiendo que el flujo migratorio y la propensión de la población española a emigrar al extranjero se mantiene constante a los niveles de 2015, en España habría en el año 2020 unos 115000 habitantes más procedentes de los intercambios de población con el extranjero y esta tendencia positiva en las migraciones se mantendría e incluso reforzaría en años sucesivos llegando a alcanzar los 3.1 millones de personas inmigrantes en el año 2066.

En resumen, las proyecciones de los principales motores demográficos ponen de manifiesto que el fenómeno del envejecimiento, lejos de estancarse, tiene visos de seguir aumentando con el tiempo y las perspectivas de migración no resultan suficientes para frenar esta dinámica. Este aumento de la longevidad de las personas conlleva, en primer lugar, un replanteamiento del sistema de pensiones, para adaptarlo a una esperanza de vida creciente, así como la asunción de cambios en ámbitos médicos, sociales, psicológicos y económicos, en los que el envejecimiento también produce multitud de efectos.

\section{BIBLIOGRAFÍA}

Abellán García, A. y Ayala García, A. (2017). Un perfil de las personas mayores en España, 2017. Indicadores estadísticos básicos. Consejo Superior de Investigaciones Científicas (CSIC). Centro de Ciencias Humanas y Sociales (CCHS). Envejecimiento en red. Disponible en: http://envejecimiento.csic.es/documentos/ documentos/enred-indicadoresbasicos17.pdf

Ayuso, M. y R. Holzmann (2014a). Longevidad: un breve análisis global y actuarial. Documento de Trabajo $n^{0} 1 / 2014$, Instituto BBVA de Pensiones, Madrid. Disponible en: https://www.jubilaciondefuturo.es/recursos/doc/pensiones/20131003/ posts/2015-1-longevidad-un-breve-analisis-global-y-actuarial-esp.pdf

Ayuso, M. y R. Holzmann (2014b). Condicionantes demográficos, estructuras de población y sistemas de pensiones. Documento de Trabajo ${ }^{\circ} 5 / 2014$, Instituto BBVA de Pensiones, Madrid. Disponible en: https://www.jubilaciondefuturo.es/ recursos/doc/pensiones/20131003/posts/2015-5-condicionantes-demograficosestructuras-de-poblacion-y-sistemas-de-pensio.pdf 
Ayuso, M. y R. Holzmann (2014c). Natalidad, pirámide poblacional y movimientos migratorios en España: su efecto en el sistema de pensiones. Documento de Trabajo $n^{\circ} 8 / 2014$, Instituto BBVA de Pensiones, Madrid. Disponible en: https://www. jubilaciondefuturo.es/recursos/doc/pensiones/20131003/posts/2015-8-natalidadpiramide-poblacional-y-movimientos-migratorios-en-espana-esp.pdf

INE (2017a). Indicadores demográficos básicos. Instituto Nacional de Estadística. Madrid. Disponible en: http://www.ine.es/metodologia/t20/metodologia_idb.pdf

INE (2016a). Cifras de población a 1 de enero de 2016. Estadística de migraciones. 2015. Adquisiciones de nacionalidad española de residentes 2015. Notas de prensa del Instituto Nacional de Estadística. Madrid. Disponible en: http://www. ine.es/prensa/np980.pdf

INE (2017b). Movimiento natural de la población (nacimientos, defunciones y matrimonios). Indicadores demográficos básicos. Notas de prensa del Instituto Nacional de Estadística. Madrid. Disponible en: http://www.ine.es/prensa/mnp_2016_p.pdf

INE (2016b). Movimiento natural de la población (nacimientos, defunciones y matrimonios). Indicadores demográficos básicos. Notas de prensa del Instituto Nacional de Estadística. Madrid. Disponible en: http://www.ine.es/prensa/np976.pdf

INE (2016c). Cifras de población a 1 de julio de 2016. Estadística de migraciones. Primer semestre de 2016. Notas de prensa del Instituto Nacional de Estadística. Madrid. Disponible en: http://www.ine.es/prensa/np1010.pdf

INE (2016d). Proyecciones de Población 2016-2066. Notas de prensa del Instituto Nacional de Estadística. Madrid. Disponible en: http://www.ine.es/prensa/ np994.pdf

Lorenzo Carrascosa, L (2003). Consecuencias del envejecimiento de la población: el futuro de las pensiones. Disponible en: www.ine.es/daco/daco42/sociales/ infosoc_envej.pdf

ONU (2015). World Population Prospects: The 2015 Revision, United Nations, Department of Economic and Social Affairs, Population Division. 